\title{
Reviewers in 2013
}

(C) The Japan Society of Brain Tumor Pathology 2013

Brain Tumor Pathology Editorial Office wishes to thank the following reviewers (non-editorial board members) for their contributions in 2013.

Taku Homma, Saitama

Hiroshi Nishihara, Hokkaido

Tatsuo Sawada, Tokyo

Mishie Tanino, Hokkaido 